\title{
Doppler flow characteristics in the assessment of pulmonary artery pressure in ductus arteriosus
}

\author{
A B HOUSTON, M K LIM, W B DOIG, J GNANAPRAGASAM, E N COLEMAN, \\ M P G JAMIESON, J C S POLLOCK \\ From the Departments of Cardiology, Cardiac Surgery, and Child Health, Royal Hospital for Sick Children, \\ Glasgow
}

SUMMARY The Doppler spectral pattern of flow through the ductus arteriosus was studied in 117 patients. In 37 who underwent catheterisation, Doppler records and aortic and pulmonary artery pressure were available (21 simultaneously with two catheters) for review while the others had surgical ligation of the duct on the basis of the results of non-invasive tests. Four flow patterns were obtained: (a) continuous flow, maximum velocity in late systole with gradual fall throughout diastole; $(b)$ continuous flow, high systolic flow with rapid fall to a very low early diastolic velocity maintained throughout diastole; $(c)$ continuous low velocity, maximum in late diastole; and $(d)$ bidirectional flow. Flow pattern $(a)$ was associated with normal or slightly raised pulmonary artery pressure; $(b)$ with raised pulmonary artery pressure; and $(c)$ and $(d)$ with pulmonary artery pressure at systemic values. Comparison of the Doppler and measured pressure differences between the great arteries was reasonably good for peak values but poor for the trough readings.

Doppler ultrasound clearly showed ductal flow; the flow pattern gave an indication of the pulmonary artery pressure, but pressure measurement by application of the Bernoulli equation to the flow velocities cannot yet be regarded as reliable.

The diagnosis of ductus arteriosus is usually readily apparent on clinical examination but may be difficult where the murmur is obscured by another louder one or is unrecognisable or atypical because of pulmonary hypertension ${ }^{1}$; and echocardiography is of limited value in confirming its presence. ${ }^{2}$ In addition, where expertise in surgery of infants is not available patients may not undergo ductus ligation routinely until after the second year of life; it is then essential to be able to assess the pulmonary pressure to select those who need earlier closure. Doppler ultrasound has the potential to show and measure the velocity of ductal flow and may thus be of practical value in patient management by determining the pulmonary artery pressure or confirming the diagnosis where there is difficulty with either clinical or echocardiographic assessment.

Ductal flow can be shown with pulsed ${ }^{3}$ and continuous wave ${ }^{4}$ Doppler; and colour Doppler flow mapping ${ }^{5}$ outlines the ductus when it is difficult to image

Requests for reprints to Dr A B Houston, Royal Hospital for Sick Children, Yorkhill, Glasgow G3 8SJ.

Accepted for publication 31 March 1989 with echocardiography. In most cases a signal showing continuous flow throughout the cardiac cycle is clearly recognised, but the flow pattern may be atypical when the usual pressure differences between the great arteries do not occur. It has been reported that the flow pattern changes with pulmonary artery hypertension $^{6}$ and that the pulmonary pressure can be predicted from the application of the Bernoulli equation ${ }^{7}$ to the ductal flow velocity. ${ }^{4}$ While one recent study reported difficulties in determining the pressure drop between the great arteries ${ }^{8}$ from the Bernoulli equation, another found this to be satisfactory. ${ }^{9}$ Although these studies reported on a total of 55 individuals only six were investigated with dual catheter pressure measurements and no attention was given to the possible differences between the peak to peak and trough to trough and instantaneous maximum and minimum pressure differences.

We found that pulmonary pressure cannot always be predicted reliably and that the flow patterns may be of more value for assessing pulmonary pressure. Thus we now report our experience with Doppler ultrasound in patients with a ductus arteriosus undergoing cardiac catheterisation or surgical ligation, with particular reference to the difference 
between the instantaneous and peak to peak measured pressures, the variability in the spectral flow patterns, and the use of the spectral signal to attempt to calculate the pulmonary artery pressure by the application of the Bernoulli equation.

\section{Patients and methods}

Patients with a ductus dependent circulation such as preductal coarctation were excluded. Between August 1985 and September 1988, 159 other patients (aged two days to 14 years) with a ductus arteriosus underwent either cardiac catheterisation (40) or direct surgical closure (119) on the basis of clinical and non-invasive studies.

The Doppler spectral recordings and pulmonary arterial and aortic pressures at catheterisation were available for review in 37 children (aged two days to 15 years, mean 3.5 years). The pressures were measured through fluid filled catheters attached to strain gauge transducers that were initially matched as closely as possible with both catheters in the same chamber. Pressures were measured through two catheters (21) or by withdrawal of a single catheter (16) from the descending aorta to the main pulmonary artery. In three in whom an arterial approach was used for the second catheter the aortic pressure was measured with it in the arch; a second venous catheter was passed through the pulmonary artery into the descending aorta in the others. The Doppler study was performed simultaneously with the pressure recordings in 27 , including 20 studied with two catheters.

Of these 37, 16 had an isolated ductus arteriosus, three a recanalised ductus, seven a ventricular septal defect, two mitral valve disease, two an atrial septal defect, two peripheral pulmonary artery stenosis, one cardiomyopathy, one a right ductus arteriosus, one a pulmonary artery sling, one aortic stenosis, and one an atrioventricular canal with total anomalous pulmonary venous drainage.

Thirty of those not catheterised were preterm infants with respiratory distress syndrome, and Doppler studies were not routinely performed; they have not been considered in the analysis of the results. Doppler records were available in 80 of the 89 other patients.

Thus simultaneous Doppler and catheter data were available in 27 (20 measured with two catheters), non-simultaneous Doppler and catheter data in 10, and Doppler measurements alone in 80 undergoing operation without catheterisation. Dual catheter pressures were available in 21 , in one of whom simultaneous Doppler data were not available.

The early Doppler studies were performed with a non-imaging Alfred velocimeter (Vingmed) inter- faced to a Doptek spectrum analyser and later ones, after January 1987, with a Vingmed CFM 700 system with imaging and colour flow mapping. With imaging and colour Doppler a short axis view of the great arteries was used and the beam was aligned in the distal main pulmonary artery where ductal flow was apparent. Because the maximum velocity signal may be obtained from another site we sought it from the first, second, and third left interspaces and the suprasternal notch, using the stand alone transducer when necessary. With the non-imaging system the signal was usually obtained easily with continuous wave Doppler and the stand alone transducer by interrogating the same positions. The maximum and minimum frequency shifts were measured from the Doppler signal. From these we derived the maximum and minimum velocities $(V)$ and estimated the pressure drops $(P)$ from the modified Bernoulli formula, $P=4 V^{2}$.

Of the 117 available Doppler studies the stand alone system was used in 48 ( 25 undergoing catheterisation) and the duplex colour flow system in 69 (12 undergoing catheterisation).

The spectral tracings were analysed to review both the timing and patterns of the signals in systole and diastole. The maximum velocity in systole and the minimum velocity in diastole were then measured and the maximum and minimum pressure differences between the great arteries calculated by the modified Bernoulli equation. ${ }^{7}$ The Doppler and catheterisation pressure gradients between the great arteries were then compared for both the maximum pressure differences in systole and the minimum differences in diastole, the differences between them being analysed by the method of Bland and Altman. ${ }^{10}$ Study of the waveforms suggested that higher pulmonary artery pressure was associated with an increase in the ratio of the systolic to diastolic flow velocity. Thus in addition to measuring this ratio with Doppler, we also calculated the ratio from the measured pressure differences by translating their values in $\mathrm{mm} \mathrm{Hg}$ into $\mathrm{m} / \mathrm{s}$, as would be measured with Doppler if the Doppler and catheter gradients were exactly equal.

The pressure tracings were also analysed to measure the instantaneous maximum and minimum gradients and to compare them with peak to peak and trough to trough gradients respectively.

\section{Results}

\section{PRESSURE MEASUREMENTS AT}

\section{CATHETERISATION BY TWO CATHETERS}

Three infants in whom the differences between the aortic and pulmonary pressures was greatest in diastole when they were studied with two catheters 
(see figs 3 and 4) were excluded in this analysis. In the other 18 studied with two catheters the peak to peak gradients in systole ranged from 28 to $116 \mathrm{~mm} \mathrm{Hg}$ and instantaneous ones from 34 to $124 \mathrm{~mm} \mathrm{Hg}$. The difference was greater than $10 \mathrm{~mm} \mathrm{Hg}$ in only two (14 and $16 \mathrm{~mm} \mathrm{Hg}$ ) with mean (2 SD) of $6.7(8.3)$. Similarly the trough to trough gradients in diastole ranged from 0 to $83 \mathrm{~mm} \mathrm{Hg}$ and the instantaneous minimum from 0 to $79 \mathrm{~mm} \mathrm{Hg}$. The maximum difference was $11 \mathrm{~mm} \mathrm{Hg}$ with mean (2 SD) of 5.6 $(7 \cdot 2)$.

\section{DOPPLER FLOW PATTERNS IN CATHETERISED PATIENTS}

The flow patterns in the 37 patients showed variation in the timing of and relative values of the maximum and minimum velocities in systole and diastole. Inspection of the tracings showed four different flow patterns:

(a) Continuous flow: maximum velocity in late systole with a gradual fall throughout diastole (26 of the 37)

The ductus arteriosus signal characteristically showed continuous flow (fig 1a) with maximum velocity in late systole, ranging from 3.3 to 4.7 (mean $4 \cdot 1) \mathrm{m} / \mathrm{s}$, equivalent to a gradient of 44 to $88 \mathrm{~mm} \mathrm{Hg}$. The velocity signal then fell gradually to its lowest value just after the $R$ wave, often showing an abrupt dip before the rapid rise to its maximum. The lower velocity was still clearly discernible, being above 1.5 $\mathrm{m} / \mathrm{s}$, ranging from 1.5 to 3.5 (mean 2.5 ) $\mathrm{m} / \mathrm{s}$, equivalent to a pressure drop of $9-50 \mathrm{~mm} \mathrm{Hg}$. The ratio of systolic to diastolic velocities was from 1.3 to 2.4 (mean (SD) $1.6(0.3)$ ) (two of 2.4 others $\leqslant 2 \cdot 1$ ). These patients had a relatively high pressure difference between the great arteries throughout the cardiac cycle (fig 1a): the pulmonary artery systolic pressure was $<50 \mathrm{~mm} \mathrm{Hg}(20-30$ in six, 31-40 in 17, 41-50 in three, and the diastolic pressure was $\leqslant 30 \mathrm{~mm} \mathrm{Hg}(0$ 10 in five, $11-20$ in $15,21-30$ in six).

(b) Continuous flow: high systolic velocity with rapid fall to a very low early diastolic velocity that was maintained throughout diastole (seven of the 37)

The systolic signal was of relatively high velocity (equivalent to a pressure difference of $16-50 \mathrm{~mm} \mathrm{Hg}$ ) falling rapidly at end systole to a relatively low velocity $(<1.0 \mathrm{~m} / \mathrm{s})$ that was maintained throughout diastole (fig 2a). The measured pressures in diastole in the aorta and pulmonary artery were virtually equal (fig 2b). Pulmonary systolic pressures were all $>50 \mathrm{~mm} \mathrm{Hg}(52-88 \mathrm{~mm} \mathrm{Hg})$ and the diastolic ones above $30 \mathrm{~mm} \mathrm{Hg}(32-51 \mathrm{~mm} \mathrm{Hg})$. The ratio of systolic to diastolic velocities ranged from $2 \cdot 0$ to 5.0 (mean (SD) $3.4(1.1)$ ) (one of 2.0 others $\geqslant 2.9$ ).

Consideration of the 33 with flow patterns $a$ and $b$

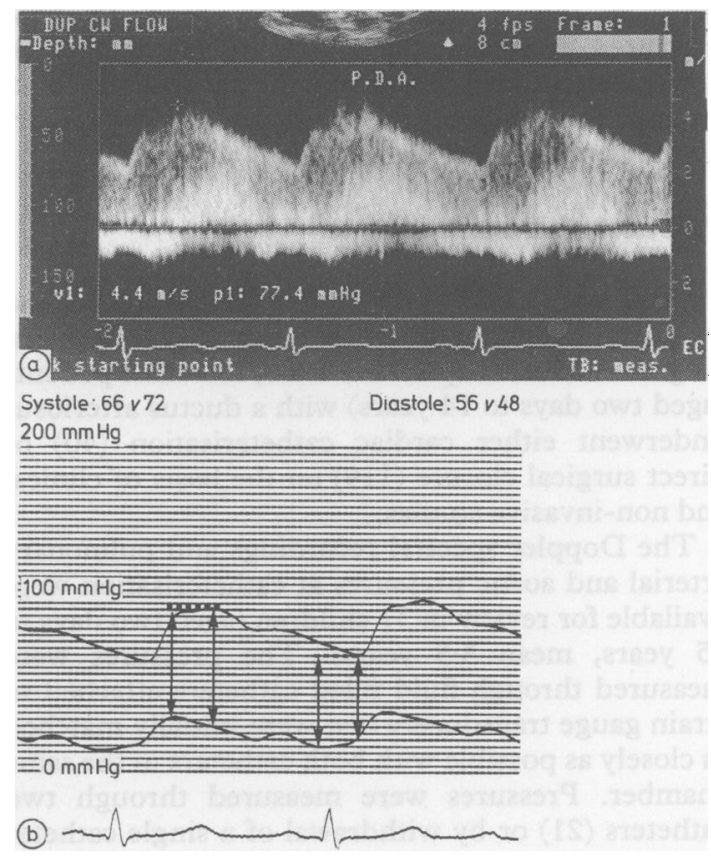

Fig 1 Records from a patient with normal pulmonary artery pressure. The continuous wave (CW) Doppler signal (a) shows velocity reaching a maximum in late systole and falling to a minimum just after the $R$ wave. The pressure record $(b)$ shows that the pressure difference between the arteries mirrors this pattern and remains relatively large throughout the cardiac cycle. Note the difference between the instantaneous maximum and minimum gradients and the peak to peak and trough to trough values.

showed that all but one of the 27 with a ratio of $<2.5$ had a pulmonary pressure of $<50 \mathrm{~mm} \mathrm{Hg}$ in systole and $30 \mathrm{~mm} \mathrm{Hg}$ in diastole, and all six with a ratio $>2.8$ had pressures $>50$ and $30 \mathrm{~mm} \mathrm{Hg}$ during systole and diastole respectively. When the catheter gradients in $\mathrm{mm} \mathbf{~ H g}$ were translated into velocity in $\mathrm{m} / \mathrm{s}$ and the ratio calculated it was $<1.6$ in all with pulmonary pressure $<50 / 30 \mathrm{~mm} \mathrm{Hg}$ and higher than this in the seven with higher pulmonary pressure $(1 \cdot 7,1 \cdot 9,2 \cdot 5,2 \cdot 9,3 \cdot 1$, and infinity in two).

\section{(c) Continuous low velocity flow, maximum in late diastole}

In one infant with a ventricular septal defect and ductus arteriosus the spectral signal showed continuous low velocity flow but with the maximum velocity $(1.6 \mathrm{~m} / \mathrm{s})$ late in diastole and the minimum one at end systole (fig $3 \mathrm{a}$ ). Simultaneously measured pressures (fig $3 \mathrm{~b}$ ) showed a raised pulmonary pressure $(60 / 20 \mathrm{~mm} \mathrm{Hg})$ with the pressure difference between the great arteries altered such that it was 


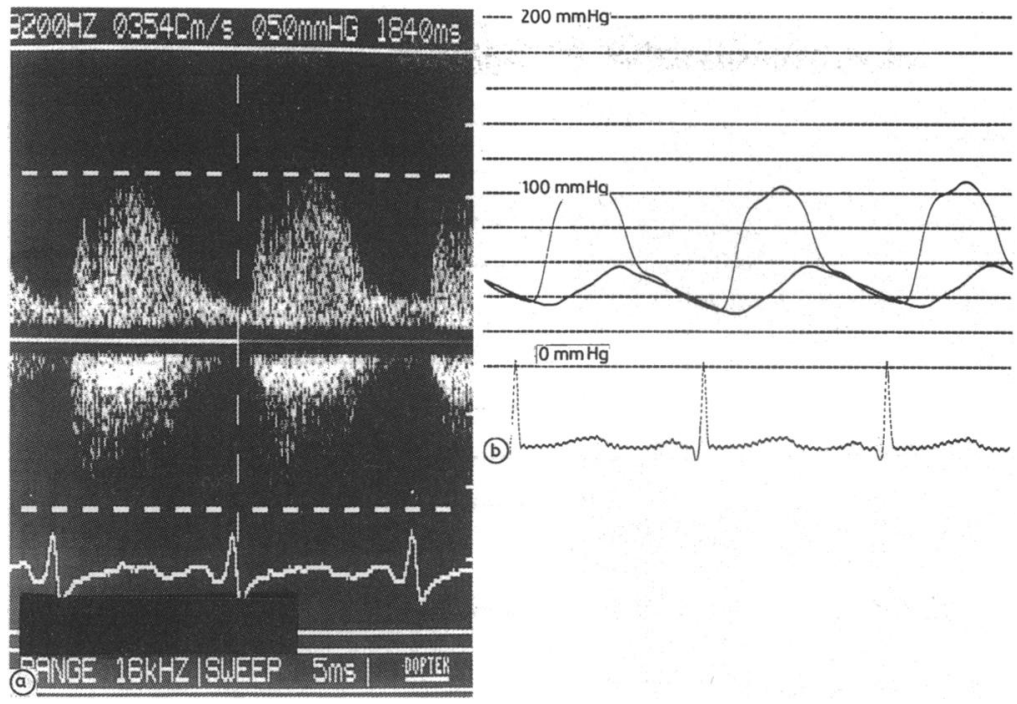

Fig 2 Records from a patient with raised pulmonary artery pressure. The signal of continuous wave Doppler (a) shows maximum velocity in late systole and falls rapidly to a relatively low diastolic velocity, which also reaches its minimum just after the $R$ wave. The pressure record (b) shows that the pressure difference between the arteries mirrors this pattern being high in systole but virtually absent in diastole.

greatest in late diastole and early systole, and least in mid to late systole.

\section{(d) Bidirectional flow}

In three infants with pulmonary artery pressure at approximately systemic values the Doppler signal showed bidirectional flow-from the pulmonary artery to the aorta in systole and in the opposite direction in diastole. At catheterisation two had pulmonary artery hypertension (equivalent to systemic pressure) with ventricular septal defects (multiple in one) and large left to right shunts. Doppler showed flow from the pulmonary artery to the aorta for a short time in systole, with aorta to pulmonary artery flow throughout the rest of the cardiac cycle (fig 4a) and in the infant studied with two catheters simultaneous pressure recordings (fig 4 b) showed that pressure in the pulmonary artery was higher than in the aorta for a short time in mid systole; pressure in the aorta was higher for the rest of the cardiac cycle. In the third, a two day old infant with dilated cardiomyopathy catheterised to exclude an anomalous coronary artery, flow from the pulmonary artery to aorta measured by Doppler lasted almost as long as flow from the aorta to pulmonary artery. The pressure tracing during withdrawal of the catheter indicated that the pulmonary artery pressure was higher in systole and lower in diastole than that in the aorta; this Doppler appearance is similar to that in other patients with persistent fetal circulation who cannot be included in this series.

\section{DOPPLER WAVEFORMS IN PATIENTS WHO WERE NOT CATHETERISED}

Of the 80 patients in whom Doppler studies were available the waveform showed a slow fall with a relatively high diastolic velocity in 71 (as in fig 1), while eight showed a sharp fall with a low velocity throughout diastole (as in fig 2) and one showed bidirectional flow.

\section{COMPARISON OF DOPPLER AND SIMULTANEOUS MEASURED GRADIENTS}

The three infants with pulmonary to aortic flow in systole (fig 4) were excluded in this assessment, leaving a total of 24 patients studied at catheterisation. In 18 of those dual catheter studies were performed and instantaneous gradients could be measured.

The method of Bland and Altman ${ }^{10}$ showed that the difference between the Doppler and peak to peak maximum gradients $(n=24)$ ranged from -20 to 22 $\mathrm{mm} \mathrm{Hg}$ with mean (2SD) of $1.6(24 \cdot 1) \mathrm{mm} \mathrm{Hg}$ (fig 5, top), while the difference between the minimum and trough values ranged from -40 to +9 , mean -13.6 (24.9) $\mathrm{mm} \mathrm{Hg}$ (fig 5, bottom). When Doppler values were compared with instantaneous maximum values $(n=18)$ the mean $(2$ SD) was $-7.9(30.6)$ and 


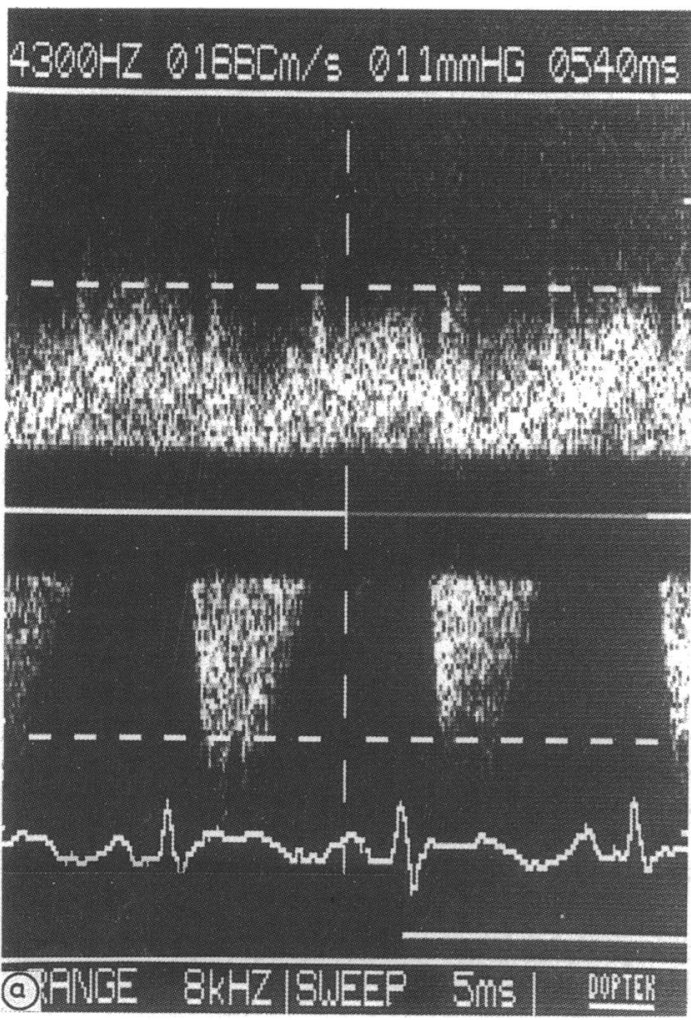

compared with instantaneous minimum values it was $-11 \cdot 3(28 \cdot 1)$.

\section{Discussion}

We found that Doppler ultrasound always detected ductal flow. It is important to be aware of the different waveforms found when pulmonary pressure is abnormal, because patients with an atypical murmur have the less common waveforms, and it is these patients in whom clinical examination may be uncertain. The most common signal (fig 1 ) is continuous, but the signal may have a sharp late systolic fall with a very low velocity throughout diastole (fig 2 ), a higher diastole than systolic velocity (fig 3), or show bidirectional flow (fig 4). The continuous signal (type a) is likely to be associated with a low or moderately raised pulmonary pressure (here systolic $<50 \mathrm{~mm} \mathrm{Hg}$ and diastolic $<30 \mathrm{~mm} \mathrm{Hg}$ ) and the signal with sharp fall at end systole (type b) with a higher pressure. This distinction is not always an obvious one, is to an extent subjective, and does not allow a clear differentiation; a type b signal may simply represent a non-restrictive ductus. A signal showing a velocity higher in diastole than systole (type c) or bidirectional flow (type d) indicates 


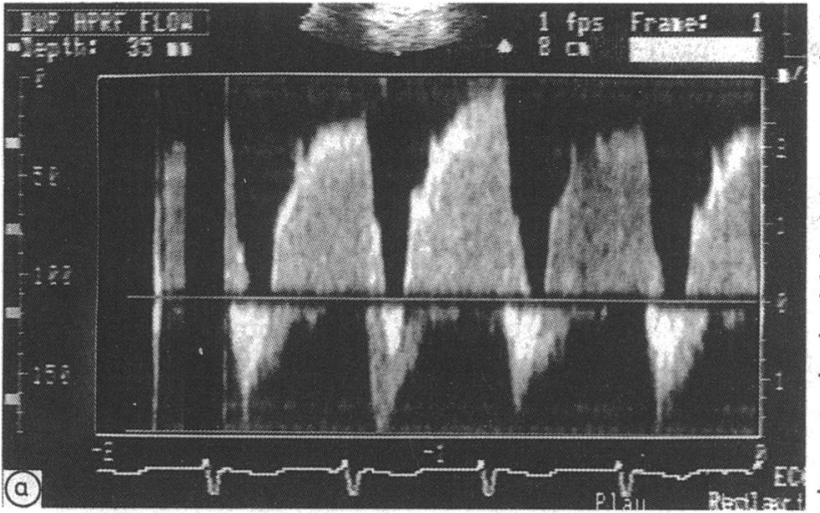

$100 \mathrm{~mm} \mathrm{Hg}$

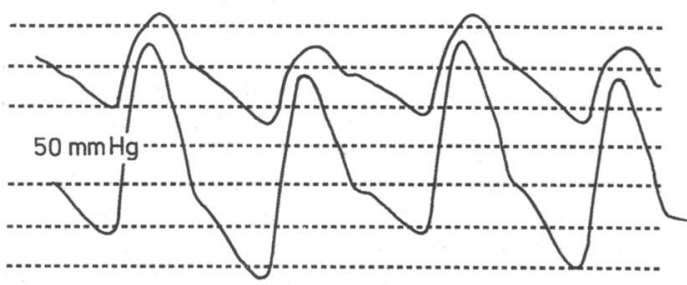

O

the aorta to the pulmonary artery and those with bidirectional shunting. ' Spach et al did not comment on the pressure differences throughout the cardiac cycle between those with moderately raised and normal pulmonary artery pressure ${ }^{11}$ and the differential suggested in this report is somewhat subjective. The ratio of maximum to minimum pressure gradient does not allow a clear distinction between high and low pulmonary artery pressure but it may be useful to regard a ratio of $\geqslant 2.5$ as suggesting an important increase in pulmonary pressure although a lower one does not exclude an increased pulmonary pressure. Although the catheter studies showed a similar variation, a ratio of $\leqslant 1.6$ was associated with lower pulmonary pressure. In addition, calculation of this ratio in the 26 measurements in infants with a ductus arteriosus reported by Rudolph et al $^{1}$ does not support this contention. This ratio is likely to be related to more complex haemodynamic findings than simply pulmonary artery pressure and further study is necessary to ascertain the true value of this ratio in the assessment of pulmonary artery pressure.

The presence of pulmonary artery pressure at systemic values produces a different signal, possibly with bidirectional shunting. Musewe et al reported that where there was continuous left to right shunting

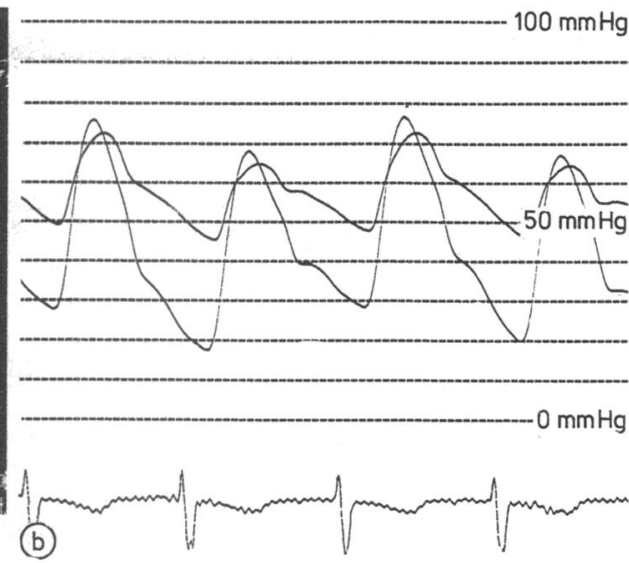

Fig 4 Records from a patient with pulmonary hypertension and bidirectional shunting through the ductus. The high pulse repetition frequency Doppler signal (a) shows bidirectional flow while the simultaneous pressure record ( $b$ ) shows that this corresponds to pressure differences between the great arteries. Modification of the pressure tracing (c) by plotting the aortic pressure $10 \mathrm{~mm} \mathrm{Hg}$ higher gives a tracing similar to fig $3 b$.

Doppler velocity reached a maximum later in the cardiac cycle with increasing pulmonary artery pressure'; the example in fig 3a presumably represents the extreme end of this, being maximum at the onset of the QRS complex. Although the waveforms in figs 3 and $4 \mathrm{~b}$ initially seem different, study of the pressure signals shows that they both reflect similar pulmonary artery pressures, which are approximately equal to the aortic ones in systole and much less in diastole. Thus if the aortic pressure from fig $4 \mathrm{~b}$ (which was described by Spach et al in patients with bidirectional pressure gradient and flow $^{11}$ ) is plotted about $10 \mathrm{~mm} \mathrm{Hg}$ higher (fig 4c) it gives a record similar to fig 3b. Patterns $c$ and $d$ are indications that the pulmonary pressure is approximately at systemic values, with $d$ being the more severe, perhaps indicating increasing resistance.

It has been suggested that because the aortic pressure can be measured with a sphygmomanometer the Doppler gradient between the great arteries can be used to assess the pulmonary pressure in the ductus arteriosus. ${ }^{49}$ This study, like that reported by Hiraishi et al, ${ }^{8}$ found considerable variability between the measured gradients and Doppler gradients in both systole and diastole. Because those with very high pulmonary pressure 


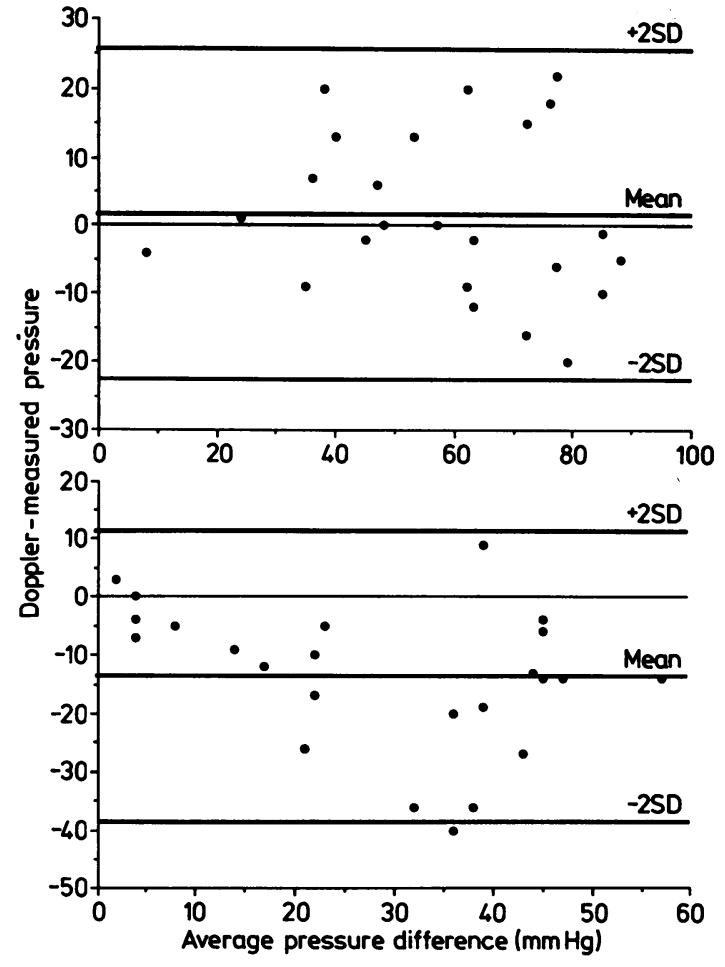

Fig 5 Comparison of the difference in the Doppler and measured peak to peak (top) and trough to trough (bottom) pressures against the average of the measurements made simultaneously at the time of catheterisation.

and abnormal flow were excluded from the analysis, this group is biased towards those with a relatively high pressure difference. But the results show both overestimation and underestimation, and in our hands the technique cannot yet be relied upon. Pressure underestimation may be the result of difficulty in aligning the Doppler beam and flow direction and many studies were performed without colour Doppler guidance. Furthermore, the transducer was adjusted to show the highest velocity systolic signal and the systolic and diastolic velocities were measured from it; it may be that an alteration in the flow direction in diastole resulted in a considerable underestimation of the diastolic gradient and particularly poor correlation in this study. In addition in some cases the spectral signal was of low intensity and this may be a further source of error.

This study indicates that Doppler ultrasound, stand alone spectral as well as duplex colour flow mapping, is a reliable means of showing the presence of a ductus arteriosus. It also suggests that Doppler may not accurately estimate the pulmonary artery pressure from the ductal flow signal. If Doppler is used to assess the pulmonary pressure we suggest that the flow pattern should be considered in addition to calculating the absolute pressures from the flow velocities. We conclude that the clinical accuracy and value of Doppler ultrasound in relation to other noninvasive means of assessing the pulmonary pressure are as yet unproved.

JG is supported by a grant from the Equipment Evaluation Committee of the Scottish Home and Health Department.

\section{References}

1 Rudolph AM, Mayer FE, Nadas AS, Gross RE. Patent ductus arteriosus. A clinical and hemodynamic study of 23 patients in the first year of life. Pediatrics 1958;22:892-903.

2 Wilson N, Dickinson DF, Goldberg SJ, Scott O. Pulmonary artery velocity patterns in ductus arteriosus. Br Heart $J$ 1984;52:462-4.

3 Stevenson JG, Kawabori I, Guntheroth WG. Pulsed Doppler echocardiographic diagnosis of patent ductus arteriosus: sensitivity, specificity, limitations and technical features. Cathet Cardiovasc Diagn 1980;6: 255-63.

4 Hatle L, Angelsen B. Doppler ultrasound in cardiology: physical principles and clinical applications. Philadelphia: Lea and Febiger, 1985:220-8.

5 Swensson RE, Valder-Cruz LM, Sahn DJ, et al. Realtime Doppler color flow mapping for detection of patent arterial ductus. J Am Coll Cardiol 1986;8: 1105-12.

6 Stevenson JG, Kawabori I, Guntheroth WG. Noninvasive detection of pulmonary hypertension in patent ductus arteriosus by pulsed Doppler echocardiography. Circulation 1979;60:355-9.

7 Hatle L, Brubakk A, Tromsdal A, Angelsen B. Noninvasive assessment of pressure drop in mitral stenosis by Doppler ultrasound. Br Heart $J$ 1978;40: 131-40.

8 Hiraishi S, Horiguchi Y, Misawa H, et al. Noninvasive Doppler echocardiographic evaluation of shunt flow dynamics of the ductus arteriosus. Circulation 1987; 75:1146-53.

9 Musewe NN, Smallhorn JF, Benson LN, Burrows PE, Freedom RM. Validation of Doppler-derived pulmonary arterial pressure in patients with ductus arteriosus under different hemodynamic states. Circulation 1987;76:1081-91.

10 Bland JM, Altman DG. Statistical methods for assessing agreement between two methods of clinical measurement. Lancet 1986;i:307-10.

11 Spach MS, Serwer GA, Anderson PAW, Canent RV, Levin AR. Pulsatile aortopulmonary pressure-flow dynamics of patent ductus arteriosus in patients with various haemodynamic states. Circulation 1980;61: 110-22. 